\title{
Effect of branched-chain amino acid-enriched nutritional supplementation on interferon therapy in Japanese patients with chronic hepatitis C virus infection: a retrospective study
}

Yumiko Nagao ${ }^{1 *}$, Takumi Kawaguchi ${ }^{1,2}$, Tatsuya $I_{d e}^{2}$ and Michio Sata ${ }^{1,2}$

\begin{abstract}
Background: The aims of this study were to evaluate the effects of nutritional supplementation with branched-chain amino acids (BCAA) with zinc component (Aminofee ${ }^{\circledR}$ ) on adherence to and outcome of therapy in patients treated with interferon (IFN) for chronic hepatitis $C$ and cirrhosis and to determine whether to recommend the supplement.

Methods: In this retrospective study, 51 patients who received IFN therapy were investigated among 203 consecutive patients who visited our hospital and were advised regarding the potential benefit of taking Aminofeel ${ }^{\circledR}$. Each patient was free to choose whether to purchase and take Aminofeel ${ }^{\circledR}$.

Results: Twenty four patients (group 1-A) took Aminofeel ${ }^{\circledR}$ during standard IFN therapy and 13 (group 1-B) did not. Low-dose, long-term IFN (maintenance) therapy, mainly peglated (Peg)-IFN alpha 2a, was administered to 14 patients who were difficult to treat, because of no effect or harmful side effects with standard IFN therapy, and who had advanced liver fibrosis. Among the 14, 11 patients (group 2-A) took Aminofeel ${ }^{\circledR}$ and 3 (group 2-B) did not. The prevalence of obesity was significantly higher $(P=0.04)$ in group 1-A than in group 1-B. The rate of adherence to IFN therapy was higher in group 1-A (83.3\%) than in group 1-B (53.8\%, $\mathrm{P}=0.05)$. There were no significant differences between the two groups in the rates of sustained virological response (SVR) to IFN therapy. According to multivariate analysis, two factors, SVR and intake of Aminofeel ${ }^{\circledR}$, were associated with successful adherence to IFN therapy. The adjusted odds ratios for these two factors were 13.25 and 12.59, respectively, and each was statistically significant. The SVR rate of maintenance IFN therapy was in 18.2\% group 2-A and 0\% in group 2-B.
\end{abstract}

Conclusion: Our data show that BCAA intake is useful for adherence to and effect of IFN therapy for patients with chronic hepatitis C. Nutritional supplementation with BCAA seems to be useful for HCV-infected patients receiving IFN therapy because it is impossible to introduce standard treatment for all patients among Japan's aging population.

Keywords: Branched-chain amino acids (BCAA), Interferon (IFN), Hepatitis C virus (HCV), Standard IFN therapy, Low-dose long-term IFN therapy

\footnotetext{
* Correspondence: nagao@med.kurume-u.ac.jp

'Department of Digestive Disease Information \& Research, Kurume University

School of Medicine, Kurume, Fukuoka 830-0011, Japan

Full list of author information is available at the end of the article
} 


\section{Background}

It has been estimated that between 1 and 2 million Japanese people are chronically infected with hepatitis $C$ virus $(\mathrm{HCV})$. In Japan, elderly patients are at a higher risk for hepatocellular carcinoma (HCC) but eradication of hepatitis $\mathrm{C}$ virus (HCV) has a reduced effect on hepatocarcinogenesis in older patients [1,2]. Therapy using interferon (IFN), an antiviral agent, reduces the rate of occurrence of $\mathrm{HCC}$ and improves the long-term prognosis $[3,4]$. In addition, long-term pegylated (Peg) IFN therapy reduces the incidence of $\mathrm{HCC}$ in patients with hepatitis $\mathrm{C}$ and cirrhosis [5].

The current standard therapy for chronic HCV infection is a combination of Peg-IFN alpha and ribavirin (RBV). Although this therapy can achieve a sustained virological response (SVR) in approximately half of treated individuals, it is associated with significant gastrointestinal, hematological, and psychiatric side effects [6-8]. The various complications of IFN therapy are common reasons for discontinuation of treatment of $\mathrm{HCV}[9,10]$.

Decreases in serum levels of branched-chain amino acids (BCAA) are often seen in patients with chronic liver diseases and these decreases lead to a decline in the production of albumin and detoxification of ammonia. Therefore, BCAAs are used for the treatment of hypoalbuminemia and hepatic encephalopathy [11,12]. More recently, it was reported that $\mathrm{BCAA}$ could restore impaired IFN signaling and inhibit $\mathrm{HCV}$ replication under conditions of malnutrition [13].

BCAA are available as a pharmaceutical agent and are indicated for "improvement of hypoalbuminemia in patients with decompensated liver cirrhosis who have hypoalbuminemia $(<3.5 \mathrm{~g} / \mathrm{dL})$ despite adequate dietary intake". For that reason, in Japan, the health insurance system is not available for administration of BCAA agent to patients with hepatitis $\mathrm{C}$ and compensated cirrhosis who receive IFN treatment. We developed a BCAA zinc component nutritional supplement (Aminofeel ${ }^{\circledR}$, Seikatsu Bunkasya Co. Inc. Tokyo, Japan), which is not a drug, after performing clinical testing on patients with hepatitis C. On March 1, 2007, Seikatsu Bunkasya Co released Aminofeel ${ }^{\circledR}$. A dose of Aminofeel ${ }^{\circledR}$ contains $5.0 \mathrm{mg}$ zinc and $3200.0 \mathrm{mg}$ BCAA [14]. Our previous clinical trial in 2006 showed that Aminofeel ${ }^{\circledR}$ is a useful therapeutic nutritional supplement for improvement of insulin resistance, hypoalbuminemia and taste disorders and showed efficacy and safety in a postmarketing surveillance study of all HCV-infected patients [14-17].

This retrospective study was conducted to assess the value of Aminofeel ${ }^{\circledR}$ in IFN therapy for patients with chronic hepatitis $\mathrm{C}$ and cirrhosis. The aim of the analysis was to determine the therapeutic effects and rate of viral eradication in patients treated with IFN therapy and to determine whether to recommend supplementation with Aminofeel ${ }^{\circledR}$.

\section{Results}

Effects of BCAA-enriched supplement and standard IFN therapy

We compared the characteristics of group 1-A and group 1-B (Table 1). The prevalence of obesity was significantly higher $(\mathrm{P}=0.04)$ in group 1 - $\mathrm{A}$ than group $1-\mathrm{B}$.

The rate of adherence to IFN therapy was $83.3 \%(20 / 24)$ in group 1-A patients, and $53.8 \%(7 / 13)$ in group $1-\mathrm{B}$ and was significantly higher $(\mathrm{P}=0.05)$ in group 1 -A. The average total intake Aminofeel ${ }^{\circledR}$ in group 1-A was $1,000.0 \pm$ $673.0 \mathrm{~g}$. The most effective of the treatments in group 1-A and $\mathrm{B}$ was combination therapy with Peg-IFN alpha $2 \mathrm{~b}$ and RBV. There were no significant differences in the SVR rates of IFN therapy between the two groups.

Table 2 documents the reasons for discontinuation of therapy. Adverse events and abnormal laboratory tests were documented in 6 and 3 patients, respectively. One patient was lost because of relocation. As adverse events, encephalopathy, exacerbation of hypothyroidism, fundal hemorrhage, pneumonia, anorexia, arthralgia, and sleeplessness were reported. The abnormal laboratory tests were elevated transaminases and HCV RNA levels. Seven of the 10 patients (70\%) who discontinued IFN therapy did not achieve SVR. The HCV genotype of two patients was $2 \mathrm{a}$, among three patients who achieved SVR in spite of discontinuing therapy.

\section{Multivariate analysis}

All variables in the univariable analyses were included in multivariable analysis. According to multivariate analysis, two factors, SVR and intake of Aminofeel ${ }^{\circledR}$, were associated with successful completion of IFN therapy. The adjusted odds ratios for these two factors were 13.25 and 12.59 , respectively, and each was statistically significant.

\section{Effects of BCAA-enriched supplement and maintenance IFN therapy}

We compared the characteristics of group 2-A and group 2-B (Table 3). The SVR rate of long-term IFN therapy was in $18.2 \%(2 / 11)$ group 2 -A patients and $0 \%$ $(0 / 3)$ in group $1-B$. The most useful treatment in group 2-A and B was Peg-IFN alpha 2a monotherapy.

One of two subjects in whom SVR was obtained was a 58-year-old woman who suffered from HCV \& hepatitis $B$ virus (HBV)-related liver cirrhosis. The number of PLT before IFN therapy was $4.2 \times 10^{4} / \mu \mathrm{L}$. We administered IFN beta (3 million units/day for 2 week and thereafter 3 million units/week) from September 5, 2006, and changed IFN beta to Peg-IFN alpha $2 \mathrm{~b}$ and RBV from February 8, 2007 and to Peg-IFN alpha 2a (45 $\mu \mathrm{g}$ 
Table 1 Characteristics of the study population 1 (standard IFN therapy, $n=37$ )

\begin{tabular}{|c|c|c|c|c|c|c|}
\hline & & $\begin{array}{l}\text { Group 1-A with intake } \\
\text { of Aminofeel }\end{array}$ & & $\begin{array}{l}\text { Group 1-B without } \\
\text { intake of Aminofeel }\end{array}$ & & $P$ value \\
\hline No. subjects & $n$ & 24 & & 13 & & \\
\hline Sex & male/female & $9 / 15$ & & $6 / 7$ & & NS \\
\hline Age & (mean $\pm S D)$, years & $62.3 \pm 5.5$ & & $57.5 \pm 12.6$ & & NS \\
\hline Age range & years & $50-74$ & & $26-75$ & & \\
\hline $\begin{array}{l}\text { IFN therapy for the first } \\
\text { time/retreatment }\end{array}$ & $n$ & $14 / 10$ & & $9 / 4$ & & NS \\
\hline \multirow[t]{5}{*}{ Liver diseases } & $\mathrm{CH}-\mathrm{C}$ & 16 & $66.7 \%$ & 11 & $84.6 \%$ & NS \\
\hline & $\mathrm{CH}-\mathrm{C} \& \mathrm{CH}-\mathrm{B}$ & 1 & $4.2 \%$ & 1 & $7.7 \%$ & NS \\
\hline & $\mathrm{CH}-\mathrm{C} \&$ post HCV-related HCC & 2 & $8.3 \%$ & 0 & $0.0 \%$ & NS \\
\hline & LC-C & 1 & $4.2 \%$ & 0 & $0.0 \%$ & NS \\
\hline & LC-C \& post HCV-related HCC & 4 & $16.7 \%$ & 1 & $7.7 \%$ & NS \\
\hline \multirow[t]{2}{*}{ Liver diseases } & Only $\mathrm{CH}$ & 17 & $70.8 \%$ & 12 & $92.3 \%$ & NS \\
\hline & LC or post HCC treatment & 7 & $29.2 \%$ & 1 & $7.7 \%$ & NS \\
\hline \multirow[t]{3}{*}{ HCV genotype } & $1 \mathrm{~b}$ & 19 & $79.2 \%$ & 9 & $69.2 \%$ & NS \\
\hline & $2 a$ & 3 & $12.5 \%$ & 4 & $30.8 \%$ & NS \\
\hline & $2 b$ & 2 & $8.3 \%$ & 0 & $0.0 \%$ & NS \\
\hline \multirow[t]{2}{*}{ HCV RNA level } & High & 20 & $83.3 \%$ & 11 & $84.6 \%$ & NS \\
\hline & Low & 4 & $16.7 \%$ & 2 & $15.4 \%$ & NS \\
\hline \multirow[t]{2}{*}{ Genotype.HCV RNA level } & 1b.High & 17 & $70.8 \%$ & 9 & $69.2 \%$ & NS \\
\hline & others & 7 & $29.2 \%$ & 4 & $30.8 \%$ & NS \\
\hline \multirow[t]{6}{*}{ Extrahepatic manifestations } & Diabetes millitus (positive \%) & 8 & $33.3 \%$ & 4 & $30.8 \%$ & NS \\
\hline & Hypertensiton (positive \%) & 6 & $25.0 \%$ & 2 & $15.4 \%$ & NS \\
\hline & Hyperlipidemia (positive \%) & 2 & $8.3 \%$ & 0 & $0.0 \%$ & NS \\
\hline & Oral lichen planus (positive \%) & 0 & $0.0 \%$ & 1 & $7.7 \%$ & NS \\
\hline & Hyperthyroidism (positive \%) & 0 & $0.0 \%$ & 1 & $7.7 \%$ & NS \\
\hline & Hypothyroidism (positive \%) & 3 & $12.5 \%$ & 1 & $7.7 \%$ & NS \\
\hline Total of Aminofeel intake (g) & mean \pm SD & $1000.0 \pm 673.0$ & & 0 & & $<0.0001$ \\
\hline BMI & mean \pm SD & $23.3 \pm 3.8$ & & $22.0 \pm 1.7$ & & NS \\
\hline Obesity (BMl $\geq 25 \mathrm{~kg} / \mathrm{m}^{2}$ ) & n (\%) & 7 & $29.2 \%$ & 0 & $0.0 \%$ & 0.04 \\
\hline $\mathrm{RBC}\left(\times 10^{4} / \mu \mathrm{L}\right)$ & mean $\pm S D$ & $438.8 \pm 45.9$ & & $450.2 \pm 41.7$ & & NS \\
\hline $\mathrm{Hb}(\mathrm{g} / \mathrm{dL})$ & mean $\pm S D$ & $13.7 \pm 1.2$ & & $14.1 \pm 1.5$ & & NS \\
\hline$\overline{\mathrm{PLT}}\left(\times 10^{4} / \mu \mathrm{L}\right)$ & mean $\pm S D$ & $13.3 \pm 4.1$ & & $15.5 \pm 7.0$ & & NS \\
\hline WBC $(\mu \mathrm{L})$ & mean $\pm S D$ & $4412.5 \pm 1348.9$ & & $4853.8 \pm 1066.6$ & & NS \\
\hline PT (\%) & mean \pm SD & $86.5 \pm 17.9$ & & $92.8 \pm 6.4$ & & NS \\
\hline$\overline{\mathrm{AST}}(\mathrm{U} / \mathrm{l})$ & mean \pm SD & $59.6 \pm 25.9$ & & $57.6 \pm 36.5$ & & NS \\
\hline$\overline{\mathrm{ALT}(\mathrm{U} / \mathrm{I})}$ & mean $\pm \mathrm{SD}$ & $64.7 \pm 46.9$ & & $73.2 \pm 59.2$ & & NS \\
\hline $\mathrm{LDH}(\mathrm{U} / \mathrm{I})$ & mean \pm SD & $208.9 \pm 42.0$ & & $199.5 \pm 54.1$ & & NS \\
\hline gamma GTP (U/I) & mean $\pm S D$ & $38.4 \pm 19.5$ & & $41.8 \pm 33.1$ & & NS \\
\hline$\overline{C h E}(\mathrm{U} / \mathrm{I})$ & mean \pm SD & $206.4 \pm 87.1$ & & $262.8 \pm 71.1$ & & NS \\
\hline $\mathrm{TP}(\mathrm{g} / \mathrm{dL})$ & mean $\pm \mathrm{SD}$ & $7.7 \pm 0.5$ & & $7.6 \pm 0.4$ & & NS \\
\hline Alb (g/dL) & mean $\pm S D$ & $4.00 \pm 0.5$ & & $4.17 \pm 0.2$ & & NS \\
\hline T.Bil (mg/dL) & mean \pm SD & $1.0 \pm 0.3$ & & $0.8 \pm 0.2$ & & NS \\
\hline $\mathrm{FBS}(\mathrm{mg} / \mathrm{dL})$ & mean $\pm \mathrm{SD}$ & $117.5 \pm 43.7$ & & $99.7 \pm 14.9$ & & NS \\
\hline HbA1c (\%) & mean $\pm \mathrm{SD}$ & $5.4 \pm 0.7$ & & $5.7 \pm 1.0$ & & NS \\
\hline $\mathrm{TC}(\mathrm{mg} / \mathrm{dL})$ & mean $\pm S D$ & $167.9 \pm 26.6$ & & $170.4 \pm 13.4$ & & NS \\
\hline AFP (ng/dL) & mean $\pm S D$ & $8.1 \pm 11.5$ & & $5.5 \pm 3.0$ & & NS \\
\hline IRI $(\mu \mathrm{U} / \mathrm{mL})$ & mean $\pm S D$ & $31.3 \pm 35.6$ & & $13.2 \pm 7.1$ & & NS \\
\hline
\end{tabular}


Table 1 Characteristics of the study population 1 (standard IFN therapy, $\mathbf{n = 3 7 )}$ (Continued)

\begin{tabular}{|c|c|c|c|c|c|c|}
\hline HOMA-beta & & $216.2 \pm 11.9$ & & $203.8 \pm 258.8$ & & NS \\
\hline HOMA-IR & & $11.9 \pm 21.4$ & & $3.3 \pm 2.0$ & & NS \\
\hline $\mathrm{Zn}(\mu \mathrm{g} / \mathrm{dL})$ & mean $\pm S D$ & $68.1 \pm 11.5$ & & $73.9 \pm 11.4$ & & NS \\
\hline \multirow{7}{*}{$\begin{array}{l}\text { Course of IFN } \\
\text { therapy }\end{array}$} & Peg-IFN alpha 2b/RBV & 21 & $87.5 \%$ & 8 & $61.5 \%$ & \\
\hline & Peg-IFN alpha 2a/RBV & 0 & $0.0 \%$ & 1 & $7.7 \%$ & \\
\hline & Peg-IFN alpha 2a monotherapy & 0 & $0.0 \%$ & 3 & $23.1 \%$ & \\
\hline & $\begin{array}{l}\text { Peg-IFN alpha 2a monotherapy - } \\
\text { (change) - Peg-IFN alpha 2a/RBV }\end{array}$ & 0 & $0.0 \%$ & 1 & $7.7 \%$ & \\
\hline & $\begin{array}{l}\text { Peg-IFN alpha 2a monotherapy - } \\
\text { (change) - IFN beta }\end{array}$ & 1 & $4.2 \%$ & 0 & $0.0 \%$ & \\
\hline & $\begin{array}{l}\text { Peg-IFN alpha 2b/RBV - (change) - Peg-IFN } \\
\text { alpha 2a monotherapy - (change) - } \\
\text { Peg-IFN alpha 2b/RBV }\end{array}$ & 1 & $4.2 \%$ & 0 & $0.0 \%$ & \\
\hline & IFN alpha & 1 & $4.2 \%$ & 0 & $0.0 \%$ & \\
\hline \multirow{2}{*}{$\begin{array}{l}\text { Continuation of } \\
\text { IFN therapy }\end{array}$} & Successful continuation & 20 & $83.3 \%$ & 7 & $53.8 \%$ & 0.05 \\
\hline & Discontinuation & 4 & $16.7 \%$ & 6 & $46.2 \%$ & 0.05 \\
\hline \multirow{2}{*}{$\begin{array}{l}\text { Effect of IFN } \\
\text { therapy }\end{array}$} & SVR & 12 & $50.0 \%$ & 9 & $69.2 \%$ & NS \\
\hline & Non-SVR & 12 & $50.0 \%$ & 4 & $30.8 \%$ & NS \\
\hline
\end{tabular}

$\mathrm{HCV}$, hepatitis $\mathrm{C}$ virus; $\mathrm{CH}-\mathrm{C}$, chronic hepatitis $\mathrm{C} ; \mathrm{CH}-\mathrm{B}$, chronic hepatitis $\mathrm{B}$; $\mathrm{LC}-\mathrm{C}$, liver cirrhosis type $\mathrm{C} ; \mathrm{HCC}$, hepatocellular carcinoma; $\mathrm{RBC}$, red blood cell; $\mathrm{Hb}$, hemoglobin; PLT, platelets; WBC, white blood cell; PT, prothrombin time; AST, aspartate aminotransferase; ALT, alanine aminotransferase; LDH, lactate dehydrogenase; gamma GTP, gamma-glutamyltransferase; ChE, cholinesterase; TP, total protein; Alb, albumin; T.Bil, total bilirubin; FBS, fasting blood glucose; TC, total; cholesterol; IRI, immunoreactive insulin; Zn, zinc; SVR, sustained virological response; NS, not significant; IFN, interferon; RBV, ribavirin.

Table 2 Reasons for discontinuation of standard IFN therapy $(n=10)$

\begin{tabular}{|c|c|c|c|c|c|c|c|c|c|}
\hline & Age & Sex & $\begin{array}{l}\text { Liver } \\
\text { disases }\end{array}$ & $\begin{array}{l}\text { Extrahepatic } \\
\text { manifestations }\end{array}$ & $\begin{array}{l}\mathrm{HCV} \\
\text { genotype }\end{array}$ & $\begin{array}{l}\text { HCV } \\
\text { RNA } \\
\text { level } \\
\end{array}$ & $\begin{array}{l}\text { Course of IFN } \\
\text { therapy }\end{array}$ & $\begin{array}{l}\text { Reasons for } \\
\text { discontinuation } \\
\text { of IFN therapy }\end{array}$ & $\begin{array}{l}\text { Effect of } \\
\text { IFN therapy }\end{array}$ \\
\hline \multirow[t]{4}{*}{$\begin{array}{l}\text { Group 1-A with } \\
\text { intake of Aminofeel } \\
(n=4)\end{array}$} & 70 & $\mathrm{~F}$ & $\begin{array}{l}\text { LC-C \& post } \\
\text { HCV-related } \\
\text { HCC }\end{array}$ & None & $1 \mathrm{~b}$ & High & Peg-IFN alpha 2b/RBV & Encephalopathy & Non-SVR \\
\hline & 67 & $F$ & $\mathrm{CH}-\mathrm{C}$ & $\begin{array}{l}\text { Hypertenstion, } \\
\text { hyperlipidemia, } \\
\text { and hypothyroidism }\end{array}$ & $1 \mathrm{~b}$ & High & Peg-IFN alpha 2b/RBV & $\begin{array}{l}\text { Exacerbation of } \\
\text { hypothyroidism }\end{array}$ & Non-SVR \\
\hline & 58 & $\mathrm{~F}$ & $\mathrm{CH}-\mathrm{C}$ & $\begin{array}{l}\text { Diabetes mellitus } \\
\text { and hypertenstion }\end{array}$ & $1 b$ & High & Peg-IFN alpha 2b/RBV & $\begin{array}{l}\text { Fundal } \\
\text { hemorrhage }\end{array}$ & Non-SVR \\
\hline & 66 & M & $\mathrm{CH}-\mathrm{C}$ & Diabetes mellitus & $1 \mathrm{~b}$ & High & Peg-IFN alpha 2b/RBV & $\begin{array}{l}\text { Increased } \\
\text { transaminase }\end{array}$ & Non-SVR \\
\hline \multirow{6}{*}{$\begin{array}{l}\text { Group 1-B without } \\
\text { intake of Aminofeel } \\
(n=6)\end{array}$} & 65 & $\mathrm{~F}$ & $\mathrm{CH}-\mathrm{C}$ & None & $1 b$ & High & Peg-IFN alpha 2b/RBV & $\begin{array}{l}\text { Increased HCV } \\
\text { RNA levels }\end{array}$ & Non-SVR \\
\hline & 26 & $\mathrm{~F}$ & $\mathrm{CH}-\mathrm{C}$ & None & $2 a$ & High & Peg-IFN alpha 2b/RBV & Relocation & SVR \\
\hline & 65 & $F$ & $\mathrm{CH}-\mathrm{C}$ & Hypertenstion & $1 \mathrm{~b}$ & High & Peg-IFN alpha 2b/RBV & Pneumonia & Non-SVR \\
\hline & 59 & $\mathrm{~F}$ & $\mathrm{CH}-\mathrm{C}$ & Hypertenstion & $1 \mathrm{~b}$ & High & $\begin{array}{l}\text { Peg IFN alpha } 2 \text { a } \\
\text { monotherapy - (change) - } \\
\text { Peg-IFN alpha 2a/RBV }\end{array}$ & $\begin{array}{l}\text { Increased HCV } \\
\text { RNA levels }\end{array}$ & Non-SVR \\
\hline & 54 & M & $\mathrm{CH}-\mathrm{C}$ & Diabetes mellitus & $2 a$ & Low & $\begin{array}{l}\text { Peg-IFN alpha 2a } \\
\text { monotherapy }\end{array}$ & Anorexia & SVR \\
\hline & 64 & M & $\mathrm{CH}-\mathrm{C}$ & None & $1 \mathrm{~b}$ & High & Peg-IFN alpha 2b/RBV & $\begin{array}{l}\text { Arthralgia and } \\
\text { sleeplessness }\end{array}$ & SVR \\
\hline
\end{tabular}


Table 3 Characteristics of the study population 2 (maintenance IFN therapy, $n=14$ )

\begin{tabular}{|c|c|c|c|c|c|c|}
\hline & & $\begin{array}{l}\text { Group 2-A with intake } \\
\text { of Aminofeel }\end{array}$ & & $\begin{array}{l}\text { Group 2-B without } \\
\text { intake of Aminofeel }\end{array}$ & & $P$ value \\
\hline No. subjects & $\mathrm{n}$ & 11 & & 3 & & \\
\hline Sex & male/female & $3 / 8$ & & $3 / 0$ & & 0.02 \\
\hline Age & (mean $\pm \mathrm{SD})$, years & $65.2 \pm 5.9$ & & $64.3 \pm 6.0$ & & NS \\
\hline Age range & years & $56-73$ & & $58-70$ & & \\
\hline $\begin{array}{l}\text { IFN therapy for the first } \\
\text { time/retreatment }\end{array}$ & $n$ & $5 / 6$ & & $0 / 3$ & & NS \\
\hline \multirow[t]{6}{*}{ Liver diseases } & $\mathrm{CH}-\mathrm{C}$ & 1 & $9.1 \%$ & 0 & $0.0 \%$ & \\
\hline & $\mathrm{CH}-\mathrm{C} \& \mathrm{AlH}$ & 1 & $9.1 \%$ & 0 & $0.0 \%$ & \\
\hline & $\mathrm{CH}-\mathrm{C} \&$ post HCV-related HCC & 1 & $9.1 \%$ & 1 & $33.3 \%$ & \\
\hline & LC-C & 5 & $45.5 \%$ & 0 & $0.0 \%$ & \\
\hline & LC-C \& LC-B & 1 & $9.1 \%$ & 0 & $0.0 \%$ & \\
\hline & LC-C \& post HCV-related HCC & 2 & $18.2 \%$ & 2 & $66.7 \%$ & \\
\hline \multirow[t]{2}{*}{ Liver diseases } & Only $\mathrm{CH}$ & 2 & $18.2 \%$ & 0 & $0.0 \%$ & NS \\
\hline & LC or post HCC treatmnet & 9 & $81.8 \%$ & 3 & $100.0 \%$ & NS \\
\hline \multirow[t]{3}{*}{ HCV genotype } & $1 b$ & 8 & $72.7 \%$ & 2 & $66.7 \%$ & NS \\
\hline & $2 a$ & 2 & $18.2 \%$ & 0 & $0.0 \%$ & NS \\
\hline & unknown & 1 & $9.1 \%$ & 1 & $33.3 \%$ & NS \\
\hline \multirow[t]{2}{*}{ HCV RNA level } & High & 11 & $100.0 \%$ & 3 & $100.0 \%$ & NS \\
\hline & Low & 0 & $0.0 \%$ & 0 & $0.0 \%$ & NS \\
\hline \multirow[t]{6}{*}{ Extrahepatic manifestations } & Diabetes millitus (positive \%) & 5 & $45.5 \%$ & 1 & $33.3 \%$ & NS \\
\hline & Hypertensiton (positive \%) & 6 & $54.5 \%$ & 1 & $33.3 \%$ & NS \\
\hline & Hyperlipidemia (positive \%) & 0 & $0.0 \%$ & 0 & $0.0 \%$ & NS \\
\hline & Oral lichen planus (positive \%) & 1 & $9.1 \%$ & 0 & $0.0 \%$ & NS \\
\hline & Hyperthyroidism (positive \%) & 2 & $18.2 \%$ & 0 & $0.0 \%$ & NS \\
\hline & Hypothyroidism (positive \%) & 1 & $9.1 \%$ & 0 & $0.0 \%$ & NS \\
\hline Total of Aminofeel intake $(\mathrm{g})$ & mean \pm SD & $2443.6 \pm 3209.3$ & & 0 & & $<0.01$ \\
\hline $\mathrm{BMl}$ & mean $\pm \mathrm{SD}$ & $24.3 \pm 2.0$ & & $24.5 \pm 3.1$ & & NS \\
\hline Obesity (BMI $\geq 25 \mathrm{~kg} / \mathrm{m}^{2}$ ) & $\mathrm{n}(\%)$ & 4 & $36.4 \%$ & 1 & $33.3 \%$ & NS \\
\hline $\mathrm{RBC}\left(\times 10^{4} / \mu \mathrm{L}\right)$ & mean $\pm S D$ & $402.3 \pm 62.3$ & & $417.3 \pm 29.5$ & & NS \\
\hline $\mathrm{Hb}(\mathrm{g} / \mathrm{dL})$ & mean \pm SD & $13.0 \pm 1.5$ & & $13.1 \pm 1.2$ & & NS \\
\hline$\overline{\operatorname{PLT}\left(\times 10^{4} / \mu \mathrm{L}\right)}$ & mean $\pm S D$ & $9.4 \pm 4.8$ & & $10.2 \pm 4.9$ & & NS \\
\hline$\overline{W B C}(\mu \mathrm{L})$ & mean \pm SD & $3809.1 \pm 1045.4$ & & $3500.0 \pm 1558.8$ & & NS \\
\hline PT (\%) & mean $\pm S D$ & $80.7 \pm 22.6$ & & $85.3 \pm 10.5$ & & NS \\
\hline$\overline{\mathrm{AST}}(\mathrm{U} / \mathrm{l})$ & mean $\pm S D$ & $83.9 \pm 44.4$ & & $73.7 \pm 8.4$ & & NS \\
\hline$\overline{\mathrm{ALT}(\mathrm{U} / \mathrm{l})}$ & mean \pm SD & $77.9 \pm 34.9$ & & $68.3 \pm 6.5$ & & NS \\
\hline $\mathrm{LDH}(\mathrm{U} / \mathrm{I})$ & mean $\pm \mathrm{SD}$ & $238.7 \pm 79.1$ & & $211.0 \pm 31.2$ & & NS \\
\hline gamma GTP (U/I) & mean \pm SD & $57.7 \pm 35.0$ & & $63.0 \pm 23.4$ & & NS \\
\hline ChE (U/I) & mean $\pm S D$ & $166.3 \pm 98.4$ & & $188.0 \pm 42.3$ & & NS \\
\hline $\mathrm{TP}(\mathrm{g} / \mathrm{dL})$ & mean \pm SD & $7.4 \pm 0.6$ & & $7.7 \pm 0.6$ & & NS \\
\hline Alb $(\mathrm{g} / \mathrm{dL})$ & mean $\pm S D$ & $3.59 \pm 0.5$ & & $3.67 \pm 0.1$ & & NS \\
\hline T.Bil (mg/dL) & mean $\pm S D$ & $1.0 \pm 0.3$ & & $1.1 \pm 0.4$ & & NS \\
\hline$\overline{F B S}(\mathrm{mg} / \mathrm{dL})$ & mean \pm SD & $112.7 \pm 18.4$ & & $93.3 \pm 24.0$ & & NS \\
\hline $\mathrm{HbA1c}(\%)$ & mean $\pm \mathrm{SD}$ & $5.6 \pm 0.8$ & & $5.4 \pm 0.9$ & & NS \\
\hline $\mathrm{TC}(\mathrm{mg} / \mathrm{dL})$ & mean $\pm \mathrm{SD}$ & $166.7 \pm 20.8$ & & $134.5 \pm 9.2$ & & NS \\
\hline$\overline{\operatorname{AFP}}(\mathrm{ng} / \mathrm{dL})$ & mean \pm SD & $33.7 \pm 70.2$ & & $11.7 \pm 3.8$ & & NS \\
\hline $\mathrm{IRI}(\mu \mathrm{U} / \mathrm{mL})$ & mean $\pm \mathrm{SD}$ & $20.8 \pm 15.1$ & & $15.6 \pm 8.8$ & & NS \\
\hline HOMA-beta & & $185.7 \pm 184.0$ & & $208.6 \pm 69.7$ & & NS \\
\hline
\end{tabular}


Table 3 Characteristics of the study population 2 (maintenance IFN therapy, $\mathbf{n = 1 4}$ ) (Continued)

\begin{tabular}{|c|c|c|c|c|c|c|}
\hline HOMA-IR & & $6.0 \pm 5.3$ & & $3.9 \pm 3.2$ & & NS \\
\hline $\mathrm{Zn}(\mu \mathrm{g} / \mathrm{dL})$ & mean $\pm S D$ & $62.8 \pm 10.7$ & & $67.0 \pm 2.8$ & & NS \\
\hline \multirow[t]{5}{*}{ Course of IFN therapy } & Peg-IFN alpha 2a monotherapy & 4 & $36.4 \%$ & 2 & $66.7 \%$ & \\
\hline & $\begin{array}{l}\text { Peg-IFN alpha 2a/RBV - (change) - } \\
\text { Peg-IFN alpha 2a monotherapy }\end{array}$ & 4 & $36.4 \%$ & 1 & $33.3 \%$ & \\
\hline & $\begin{array}{l}\text { IFN beta - (change) - Peg-IFN alpha } \\
\text { 2b/RBV - (change) - Peg-IFN } \\
\text { alpha 2a monotherapy }\end{array}$ & 1 & $9.1 \%$ & 0 & $0.0 \%$ & \\
\hline & $\begin{array}{l}\text { Peg-IFN alpha 2a monotherapy - } \\
\text { (change) - Peg-IFN alpha 2a/RBV - } \\
\text { (change) - Peg-IFN alpha 2a monotherapy }\end{array}$ & 1 & $9.1 \%$ & 0 & $0.0 \%$ & \\
\hline & $\begin{array}{l}\text { Peg-IFN alpha 2a monotherapy - } \\
\text { (change) - Peg-IFN alpha 2b/RBV }\end{array}$ & 1 & $9.1 \%$ & 0 & $0.0 \%$ & \\
\hline \multirow[t]{2}{*}{ Effect of IFN therapy } & SVR & 2 & $18.2 \%$ & 0 & $0.0 \%$ & NS \\
\hline & Non-SVR & 9 & $81.8 \%$ & 3 & $100.0 \%$ & NS \\
\hline
\end{tabular}

$\mathrm{HCV}$, hepatitis $\mathrm{C}$ virus; $\mathrm{CH}-\mathrm{C}$, chronic hepatitis $\mathrm{C}$; LC-C, liver cirrhosis type C; LC-B, chronic hepatitis type B; HCC, hepatocellular carcinoma; AlH, autoimmune hepatitis; IFN, interferon; RBC, red blood cell; Hb, hemoglobin; PLT, platelets; WBC, white blood cell; PT, prothrombin time; AST, aspartate aminotransferase; ALT, alanine aminotransferase; LDH, lactate dehydrogenase; gamma GTP, gamma-glutamyltransferase; ChE, cholinesterase; TP, total protein; Alb, albumin; T.Bil, total bilirubin; FBS, fasting blood glucose; TC, total; cholesterol; IRI, immunoreactive insulin; Zn, zinc; SVR, sustained virological response; NS, not significant; IFN, interferon; RBV, ribavirin.

with 2-week intervals) from July 25, 2007 to February 17, 2009.

The other was a 68-year-old woman who suffered from HCV-related liver cirrhosis and hyperthyroidism. The number of PLT before IFN therapy was $5.1 \times 10^{4} / \mu \mathrm{L}$. We administered her Peg-IFN alpha 2a (90 $\mu \mathrm{g} /$ week) from July 28, 2004 to July 24, 2009.

\section{Prevalence of extrahepatic manifestations}

The prevalence extrahepatic diseases was higher in group 2 than group 1 . The complications in group 1 included: diabetes mellitus (DM) $(n=12,32.4 \%)$, hypertension $(n=8,21.6 \%)$, hyperlipidemia $(n=2,5.4 \%)$, oral lichen planus $(\mathrm{n}=1,2.7 \%)$, hyperthyroidism $(\mathrm{n}=1,2.7 \%)$, and hypothyroidism $(n=4,10.8 \%)$. The complications in group 2 included: DM ( $n=6,42.9 \%)$, hypertension $(n=7,50.0 \%)$, oral lichen planus $(n=1,7.1 \%)$, hyperthyroidism $(n=2$, $14.3 \%)$, and hypothyroidism $(n=1,7.1 \%)$.

\section{Discussion}

$\mathrm{HCC}$ is one of the most common malignancies, especially in Japan. The most important risk factors of HCC are chronic hepatitis $C$ and $B$ and cirrhosis. IFN therapy has therapeutic benefits but management of therapy is required because the treatment leads to a number of side effects [6-10]. The major side effects include fatigue, influenza-like syndrome, gastrointestinal disturbances and abnormalities of laboratory tests. The rate of discontinuation of combination therapy with Peg-IFN alfa and RBV was reported to be $10-26 \%$ [6-10]. Most dose discontinuations resulted from adverse events, whereas only a minority required discontinuation due to abnormalities of laboratory tests [8]. Fried et al. demonstrated that a full dosing scheduling of both Peg-IFN and RBV permits $75 \%$ of early virological responders to achieve SVR, that dose reduction resulted in a fall to $67 \%$ and that discontinuation resulted in a loss of SVR, at $12 \%$ [7].

In this study, we showed that intake of a BCAAenriched supplement is significantly associated with the ability to adhere to standard IFN therapy. Our previous clinical evaluation of the BCAA-enriched supplement, Aminofeel $^{\circledR}$, in chronic hepatitis $C$ patients who were not receiving IFN therapy, showed improvement of fatigue, loss of appetite, jitteriness, and leg cramps [14]. Evidence produced elsewhere shows improvement of insulin resistance, hypoalbuminemia, taste disorders, ChE value, and prothrombin value [14-17]. Kawaguchi et al. suggested the following three reasons for improvement of subjective symptoms with BCAA intake: amelioration of hepatic encephalopathy, improvement of malnourishment by elevated tryptophan levels and increase of impaired cerebral blood flow [18-21]. Our previous study using single photon emission computed tomography (SPECT) showed decreased regional cerebral blood flow during IFN administration [22]. This phenomenon induces psychotic symptoms such as a depressive state.

We reported previously a strong association between hypoalbuminemia and mortality in a hyperendemic area (X town) of HCV infection in Japan [23]. Residents with hypoalbuminemia had a mortality of $68.0 \%$; dramatically higher than the rate of $12.1 \%$ among residents who had normal albumin levels. Improvement of hypoalbuminaemia should be considered for improvement of prognosis.

Combination therapy with Peg-IFN and RBV is a useful strategy for patients with hepatitis $C$ but it is 
impossible to introduce standard treatment for all patients because it is not adapted for abnormal biochemical findings or elderly patients. Liver-protective therapy, such as oral administration of ursodeoxycholic acid or intravenous injection of Stronger Neo-minophagen C $\left(\mathrm{SNMC}^{\circledR}\right)$, is commonly given to nonresponders to IFN therapy in Japan $[24,25]$. SNMC ${ }^{\circledR}$ is a glycyrrhizin preparation that has potent anti-inflammatory activity and has been used in Japan for centuries to treat allergic diseases and hepatitis. However, this agent is not considered to have any antiviral or anticancer effect [26]. We have reported the effect of IFN on alphafetoprotein (AFP) changes in 40 patients with chronic hepatitis $C$ [27]. AFP was reduced significantly more in the IFN group than the SNMC group $(\mathrm{P}=0.0034)$. Therapeutic strategies for hepatitis C, e.g., low-dose long-term IFN treatment, reduced the incidence not only of hepatocarcinogenesis but also esophageal varices during the 17-year observation period [28,29].

There are very few reports that SVR was obtained by long-term, low dose therapy for chronic hepatitis $\mathrm{C}$ [30]. However, in this study, we succeeded in eradicating $\mathrm{HCV}$ from two patients with cirrhosis using maintenance IFN treatment. $\mathrm{HCV}$ is frequently reported to complicate extrahepatic manifestations such as oral lichen planus, thyroid disease and DM [31]. Most of the 10 patients who discontinued standard IFN therapy had extrahepatic manifestations, and most of the 14 who received low-dose long-term IFN therapy also had such manifestations. These manifestations are reported to be exacerbated by IFN therapy and lead to discontinuation of therapy. These findings indicate the value of maintenance IFN therapy for patients with extrahepatic manifestations.

Amongst Japan's aging population, combined therapy of low-dose long-term IFN and administration of BCAA is thought to be a treatment to adopt enthusiastically. BCAA intake seems to be useful for HCV-infected patients.

\section{Conclusion}

In conclusion, our data show that BCAA intake is useful for continuation of IFN therapy for patients with HCV chronic liver diseases. There is great variability among subjects with liver disease and, because this is not a randomized study, further investigations are needed.

\section{Materials and methods}

A total of 203 patients with chronic liver diseases who had a checkup in Kurume University Hospital from March 1, 2007 to July 31, 2008 received advice about the therapeutic effects of, and methods of purchasing, Aminofeel ${ }^{\circledR}$. Each patient was free to decide whether to purchase and take Aminofeel ${ }^{\circledR}$. We excluded 15 patients whose uptake rates were below $70 \%$.

Among the remaining 188 patients, there were 51 who received IFN therapy. Thirty five patients took Aminofeel ${ }^{\circledR}$ and 16 did not. Thirty seven patients received IFN treatment with the aim of eradicating $\mathrm{HCV}$ and 14 received low-dose, long-term (maintenance) IFN treatment aimed at suppression of hepatocarcinogenesis. Natural IFN alpha, IFN beta or Peg-IFN alpha 2a was selected for IFN monotherapy. Combined standard therapy of Peg-IFN alpha $2 \mathrm{~b}$ or $2 \mathrm{a}$ and RBV was given for 24 to 72 weeks.

\section{Group 1 (standard IFN therapy)}

Among the 37 patients who received IFN therapy to eradicate HCV, 24 (group 1-A) took Aminofeel ${ }^{\circledR}$ during therapy and 13 (group 1-B) did not (Table 1). Group 1-A ranged in age from 50 to 74 years, with an average of $62.3 \pm 5.5$ years. Group 1-B ranged in age from 26 to 75 years, with an average of $57.5 \pm 12.6$ years.

The diagnosis of liver disease in group 1-A included: chronic hepatitis $C(n=16)$, chronic hepatitis $C$ and hepatitis B $(n=1)$, chronic hepatitis $C$ with posttreatment of HCC $(n=2)$, HCV-related liver cirrhosis $(\mathrm{n}=1)$, and HCV-related liver cirrhosis with posttreatment of HCC $(n=4)$. Those of group 1-B included: chronic hepatitis $C(n=11)$, chronic hepatitis $C$ and hepatitis $B(n=1)$, and HCV-related liver cirrhosis with post-treatment of HCC $(n=1)$.

The end-point was the achievement of the adherence to IFN and/or RBV therapy, defined as the receipt of $\geq 80 \%$ of scheduled IFN and/or RBV doses for $\geq 80 \%$ of the scheduled treatment period.

\section{Group 2 (low-dose, long-term IFN therapy)}

Low-dose long-term (maintenance) IFN, usually PegIFN alpha 2a, was administered to 14 patients who were difficult to treat, because of no effect or harmful side effects with standard IFN therapy, and who had advanced liver fibrosis (Table 3). Among the 14, 11 patients (group 2-A) took Aminofeel ${ }^{\circledR}$ during therapy and 3 (group 2-B) did not. Group 2-A ranged in age from 56 to 73 years, with an average of $65.2 \pm 5.9$ years. Group 2-B ranged in age from 58 to 70 years, with an average of $64.3 \pm 6.0$ years.

The diagnosis of liver disease in group 2-A included: chronic hepatitis $C(n=1)$, chronic hepatitis $C$ and autoimmune hepatitis $(n=1)$, chronic hepatitis $C$ with posttreatment of $\mathrm{HCC}(\mathrm{n}=1)$, HCV-related liver cirrhosis $(\mathrm{n}=5), \mathrm{HCV}$ and HBV-related liver cirrhosis $(\mathrm{n}=1)$, and $\mathrm{HCV}$-related liver cirrhosis with post-treatment of HCC $(n=2)$. Those of group 2-B included: chronic hepatitis $C$ with post-treatment of $\mathrm{HCC}(\mathrm{n}=1)$, and $\mathrm{HCV}$-related liver cirrhosis with post-treatment of $\mathrm{HCC}(\mathrm{n}=2)$. 


\section{Serological assays}

All patients were tested for red blood cell (RBC), white blood cell (WBC), platelets (PLT), hemoglobin ( $\mathrm{Hb}$ ), and insulin resistance index (IRI), zinc (Zn), fasting blood glucose (FBS) and HbA1c, and for the following liver function tests: serum alanine aminotransferase (ALT), aspartate aminotransferase (AST), lactate dehydrogenase (LDH), gamma-glutamyltransferase (gamma GTP), cholinesterase (ChE), prothrombin time (PT), total bilirubin (T. Bil), total cholesterol (TC), total protein (TP), and albumin (Alb). The formula for the HOMA-IR is: HOMA-IR $=$ FBS $\times$ fasting insulin $/ 405$. The formula for the HOMA-beta is: HOMA-beta $=360 \times$ fasting insulin/ FBS-63.

\section{Evaluation of liver diseases}

Anti-HCV was measured using a chemiluminescent enzyme immunoassay kit (Lumipulse II HCV, Fujirebio, Tokyo, Japan). HCV RNA in serum was analyzed by the Amplicor HCV test (Roche, Tokyo, Japan) up to December 6, 2007 and by quantitative PCR assay (COBAS AMPLICOR HCV MONITOR v 2.0 Test, COBAS AmpliPrep/COBAS Taq-Man HCV Test, Roche Molecular Systems, New Jersey, US) from December 7, 2007 $[32,33]$. HCV genotype was determined by polymerase chain reaction assay, using a mixture of primers for the subtype, as reported previously [34]. HBsAg was assayed using a chemiluminescent immunoassay kit (Architect, HBsAg QT, Dainabot, Tokyo, Japan). All patients were examined using hepatic ultrasonography, computed tomography, or magnetic resonance imaging in order to investigate the shape of the liver and lesions occupying the liver. Liver biopsy was performed on some patients.

\section{Evaluation of extrahepatic diseases}

IFN therapy may cause or worsen such immunological diseases or extrahepatic diseases. We examined theses diseases before IFN therapy. A diagnosis of oral lichen planus was made on the basis of clinical and/or histopathological features. Obesity was defined as a body mass index (BMI) $\geq 25 \mathrm{~kg} / \mathrm{m}^{2}$. Diagnosis of type $2 \mathrm{DM}$ was based on the American Diabetic Association (ADA) criteria of 1997 [35]. Hypertension was defined as a systolic blood pressure (SBP) of $140 \mathrm{mmHg}$ or higher or a diastolic blood pressure (DBP) of $90 \mathrm{mmHg}$ or higher, according to the criteria of JNC-VI of the International Hypertension Society [36]. Thyroid hormones such as FT3, FT4 and thyroid stimulating hormone were measured for all patients and thyroid echography examination was performed for some patients. Examination of the upper or lower gastrointestinal tract was performed on patients for whom it was deemed clinically necessary.

\section{Ethical considerations}

The retrospective study was approved by the Ethics Committee of Kurume University on August 4, 2008 (reference number: 06046) in accordance with the Declaration of Helsinki.

\section{Statistical analysis}

All data are expressed as mean \pm standard error. Differences between the two groups were analyzed using the Mann-Whitney $U$ test, Wilcoxon's test, and Fisher's exact test. Differences were judged significant for $\mathrm{p} \leq 0.05$ (twotailed). $\mathrm{P}$ value was rounded off to two decimal places. Adjusted odds ratios were calculated using logistic regression analysis. All statistical analyses were conducted using JMP Version 6 (SAS Institute, Cary, NC, USA). The level of statistical significance was defined as 0.05 .

\section{Abbreviations}

HCV: Hepatitis C virus; $\mathrm{CH}-\mathrm{C}$ : Chronic hepatitis C; $\mathrm{CH}-\mathrm{B}$ : Chronic hepatitis B; LC-C: Liver cirrhosis type C; LC-B: Liver cirrhosis type B; HCC: Hepatocellular carcinoma; PBC: Primary biliary cirrhosis; IFN: Interferon; RBV: Ribavirin; SVR: Sustained virological response; PLT: Platelets; Hb: Hemoglobin; ALT: Alanine aminotransferase; AST: Aspartate aminotransferase; LDH: Lactate dehydrogenase; ALP: Alkaline phosphatase; T.Bil: Total bilirubin; TC: Total cholesterol; TP: Total protein; Alb: Albumin.

\section{Competing interests}

The authors declare that they have no competing interests. None of author in this study holds and is applying for any patents relating to Aminofeel ${ }^{\circledR}$.

None of author has received fees, funding, or salary from Seikatsu Bunkasya Co.. Seikatsu Bunkasya Co is applying for a patent for Aminofeel ${ }^{\circledR}$.

\section{Authors' contributions}

YN carried out most of the data collection, designed the study, performed the statistical analysis, and drafted the manuscript. TK provided comments during manuscript preparation. TI contributed to data collections and clinical diagnosis. MS contributed to data analysis. All authors read and approved the final manuscript.

\section{Acknowledgements}

This study was supported in part by a Grant-in-Aid for Scientific Research (C) (No.22592354) from the Ministry of Education, Culture, Sports, Science and Technology of Japan.

\section{Author details}

${ }^{1}$ Department of Digestive Disease Information \& Research, Kurume University School of Medicine, Kurume, Fukuoka 830-0011, Japan. ${ }^{2}$ Division of Gastroenterology, Department of Medicine, Kurume University School of Medicine, Kurume Fukuoka 830-0011, Japan.

Received: 26 July 2012 Accepted: 5 November 2012 Published: 22 November 2012

\section{References}

1. Kiyosawa K, Uemura T, Ichijo T, Matsumoto A, Yoshizawa K, Gad A, Tanaka E: Hepatocellular carcinoma: recent trends in Japan. Gastroenterology 2004, 127(Suppl 1):17-26.

2. Asahina Y, Tsuchiya K, Tamaki N, Hirayama I, Tanaka T, Sato M, Yasui Y, Hosokawa T, Ueda K, Kuzuya T, Nakanishi H, Itakura J, Takahashi Y, Kurosaki $\mathrm{M}$, Enomoto N, Izumi N: Effect of aging on risk for hepatocellular carcinoma in chronic hepatitis C virus infection. Hepatology 2010, 52:518-527.

3. Yoshida H, Shiratori $Y$, Moriyama M, Arakawa Y, Ide T, Sata M, Inoue O, Yano M, Tanaka M, Fujiyama S, Nishiguchi S, Kuroki T, Imazeki F, Yokosuka O, Kinoyama S, Yamada G, Omata M: Interferon therapy reduces the risk for hepatocellular carcinoma: national surveillance program of cirrhotic and noncirrhotic 
patients with chronic hepatitis C in Japan. IHIT study group. Inhibition of hepatocarcinogenesis by interferon therapy. Ann Intern Med 1999, 131:174-181.

4. Yoshida H, Arakawa Y, Sata M, Nishiguchi S, Yano M, Fujiyama S, Yamada G, Yokosuka O, Shiratori Y, Omata M: Interferon therapy prolonged life expectancy among chronic hepatitis C patients. Gastroenterology 2002, 123:483-491.

5. Lok AS, Everhart JE, Wright EC, Di Bisceglie AM, Kim HY, Sterling RK, Everson GT, Lindsay KL, Lee WM, Bonkovsky HL, Dienstag JL, Ghany MG, Morishima C, Morgan TR, HALT-C Trial Group: Maintenance peginterferon therapy and other factors associated with hepatocellular carcinoma in patients with advanced hepatitis C. Gastroenterology 2011, 140:840-849.

6. Manns MP, McHutchison JG, Gordon SC, Rustgi VK, Shiffman M, Reindollar R, Goodman ZD, Koury K, Ling M, Albrecht JK: Peginterferon alfa-2b plus ribavirin compared with interferon alfa-2b plus ribavirin for initial treatment of chronic hepatitis C: a randomized trial. Lancet 2001, 358:958-965.

7. Fried MW, Shiffman ML, Reddy KR, Smith C, Marinos G, Gonçales FL Jr, Häussinger D, Diago M, Carosi G, Dhumeaux D, Craxi A, Lin A, Hoffman J, Yu J: Peginterferon alfa-2a plus ribavirin for chronic hepatitis $C$ virus infection. N Engl J Med 2002, 347:975-982.

8. Hadziyannis SJ, Sette H Jr, Morgan TR, Balan V, Diago M, Marcellin P, Ramadori G, Bodenheimer H Jr, Bernstein D, Rizzetto M, Zeuzem S, Pockros PJ, Lin A, Ackrill AM, PEGASYS International Study Group: Peginterferonalpha2a and ribavirin combination therapy in chronic hepatitis $\mathrm{C}$ : a randomized study of treatment duration and ribavirin dose. Ann Intern Med 2004, 140:346-355.

9. Fried MW: Side effects of therapy of hepatitis $\mathrm{C}$ and their management. Hepatology 2002, 36(Suppl 1):237-244.

10. Russo MW, Fried MW: Side effects of therapy for chronic hepatitis C. Gastroenterology 2003, 124:1711-1719.

11. Muto Y, Sato S, Watanabe A, Moriwaki H, Suzuki K, Kato A, Kato M, Nakamura T, Higuchi K, Nishiguchi S, Kumada H, Long-Term Survival Study Group: Effects of oral branchedchain amino acid granules on event-free survival in patients with liver cirrhosis. Clin Gastroenterol Hepatol 2005, 3:705-713.

12. Marchesini G, Zoli M, Dondi C, Bianchi G, Cirulli M, Pisi E: Anticatabolic effect of branched-chain amino acid-enriched solutions in patients with liver cirrhosis. Hepatology 1982, 2:420-425.

13. Honda M, Honda M, Takehana K, Sakai A, Tagata Y, Shirasaki T, Nishitani S, Muramatsu T, Yamashita T, Nakamoto Y, Mizukoshi E, Sakai Y, Yamashita T, Nakamura M, Shimakami T, Yi M, Lemon SM, Suzuki T, Wakita T, Kaneko S, Hokuriku Liver Study Group: Malnutrition impairs interferon signaling through mTOR and FoxO pathways in patients with chronic hepatitis $C$ Gastroenterology 2011, 141:128-140

14. Kawaguchi T, Nagao Y, Matsuoka H, Ide T, Sata M: Branched-chain amino acid-enriched supplementation improves insulin resistance in patients with chronic liver disease. Int J Mol Med 2008, 22:105-112.

15. Kawaguchi T, Taniguchi E, Itou M, Sumie $\mathrm{S}$, Oriishi T, Matsuoka H, Nagao $Y$, Sata M: Branched-chain amino acids improve insulin resistance in patients with hepatitis $C$ virus-related liver disease: report of two cases. Liver Int 2007, 27:1287-1292.

16. Nagao Y, Matsuoka H, Kawaguchi T, Sata M: Aminofeel ${ }^{\circledR}$ improves the sensitivity to taste in patients with HCV-infected liver disease. Med SC Monit 2010, 16:7-12

17. Nagao Y, Kawaguchi T, Kakuma T, Ide T, Sata M: Post-marketing surveillance study for efficacy and safety of Aminofeel ${ }^{\circledR}$, a branched chain amino acid-enriched supplement including zinc. J New Rem \& Clin 2011, 60:1046-1063. in Japanese.

18. Wilkinson DJ, Smeeton NJ, Watt PW: Ammonia metabolism, the brain and fatigue; revisiting the link. Prog Neurobiol 2010, 91:200-219.

19. Huang A, Fuchs D, Widner B, Glover C, Henderson DC, Allen-Mersh TG Serum tryptophan decrease correlates with immune activation and impaired quality of life in colorectal cancer. Br J Cancer 2002, 86:1691-1696.

20. Yamamoto M, Iwasa M, Matsumura K, Nakagawa Y, Fujita N, Kobayashi Y, Kaito M, Takeda K, Adachi Y: Improvement of regional cerebral blood flow after oral intake of branched-chain amino acids in patients with cirrhosis. World J Gastroenterol 2005, 11:6792-6799.

21. Kawaguchi T, Izumi N, Charlton MR, Sata M: Branched-chain amino acids as pharmacological nutrients in chronic liver disease. Hepatology 2011, 54:1063-1070
22. Sasaki M, Sara M, Ishibashi M, Uchimura N, Tanikawa K: Decreased regional cerebral blood flow during interferon therapy. Hepatol Res 1997, 9:1-8.

23. Nagao Y, Sata M: Serum albumin and mortality risk in a hyperendemic area of HCV infection in Japan. Virol J 2010, 7:375.

24. Takano S, Ito Y, Yokosuka O, Ohto M, Uchiumi K, Hirota K, Omata M: A multicenter randomized controlled dose study of ursodeoxycholic acid for chronic hepatitis C. Hepatology 1994, 20:558-564.

25. Arase Y, Ikeda K, Murashima N, Chayama K, Tsubota A, Koida I, Suzuki Y, Saitoh S, Kobayashi M, Kumada H: The long term efficacy of glycyrrhizin in chronic hepatitis C patients. Cancer 1997, 79:1494-1500.

26. van Rossum TG, Vulto AG, Hop WC, Brouwer JT, Niesters HG, Schalm SW: Intravenous glycyrrhizin for the treatment of chronic hepatitis $C$ : a double-blind, randomized, placebo-controlled phase I/II trial. J Gastroenterol Hepatol 1999, 14:1093-1099.

27. Murashima S, Tanaka M, Haramaki M, Yutani S, Nakashima Y, Harada K, Ide T, Kumashiro T, Sata M: A decrease in AFP level related to administration of interferon in patients with chronic hepatitis $C$ and a high level of AFP. Dig Dis Sci 2006, 51:808-812.

28. Nomura H, Kashiwagi $Y$, Hirano $R$, Tanimoto $H$, Tsutsumi N, Higashi M, Ishibashi H: Efficacy of low dose long-term interferon monotherapy in aged patients with chronic hepatitis $C$ genotype 1 and its relation to alpha-fetoprotein: A pilot study. Hepatol Res 2007, 37:490-497.

29. Kawaguchi T, Sumie S, Itou M, Taniguchi E, Matoba T, Sata M: Clinical benefits and cost-effectiveness of 17-year treatment with low-dose interferon-alpha $2 \mathrm{~b}$ in a patient with chronic hepatitis $\mathrm{C}$ : a case report. Dig Dis Sci 2009, 54:690-694.

30. Shima C, Yamada A, Tsubosaka M, Sakurai M, Nakatani K, Kinoshita K, Kishida O, Fujimoto T, Minami Y: A case of chronic hepatitis $C$ with sustained virological response by an intermittent use of low dose pegylated interferon. Kanzo 2008, 49:470-475. in Japanese.

31. Nagao Y, Sata M: Insulin resistance and lichen planus in patients with HCV-infectious liver diseases. J Gastroenterol Hepatol 2008, 23:580-585.

32. Lee SC, Antony A, Lee N, Leibow J, Yang JQ, Soviero S, Gutekunst K, Rosenstraus M: Improved version 2.0 qualitative and quantitative AMPLICOR reverse transcription-PCR tests for hepatitis $C$ virus RNA: calibration to international units, enhanced genotype reactivity, and performance characteristics. J Clin Microbiol 2000, 38:4171-4179.

33. Sizmann D, Boeck C, Boelter J, Fischer D, Miethke M, Nicolaus S, Zadak M, Babiel R: Fully automated quantification of hepatitis $C$ virus (HCV) RNA in human plasma and human serum by the COBAS AmpliPrep/COBAS TaqMan system. J Clin Virol 2007, 38:326-333.

34. Dusheiko G, Schmilovitz-Weiss H, Brown D, McOmish F, Yap PL, Sherlock S, Mclntyre N, Simmonds P: Hepatitis C virus genotypes: an investigation of type-specific differences in geographic origin and disease. Hepatology 1994, 19:13-18.

35. Guide ADAR: Blood glucose monitors and data management. Diabetes Forecast 2003, 2003(56):77-79.

36. The sixth report of the joint national committee on prevention, detection, evaluation, and treatment of high blood pressure. Arch Intern Med 1997, 157:2413-2446.

doi:10.1186/1743-422X-9-282

Cite this article as: Nagao et al: Effect of branched-chain amino acidenriched nutritional supplementation on interferon therapy in Japanese patients with chronic hepatitis $\mathrm{C}$ virus infection: a retrospective study. Virology Journal 2012 9:282. 